\title{
Type III Group B Streptococcal Strain Differences in Susceptibility to Opsonization with Human Serum
}

\author{
GERALD W. FISCHER, ${ }^{(26)}$ KENNETH W. HUNTER, AND SAMUEL R. WILSON \\ Uniformed Services University, Department of Pediatrics, Bethesda, Maryland, USA
}

Summary

Human serum opsonins to type III Group B streptococci (GBS) were studied in an in vitro opsonophagocytic assay. Two type III GBS test strains were susceptible (893 and IIINor) and two resistant (891 and 892 ) to opsonization by the majority of sera from 15 healthy adults. Four individuals with undetectable or low opsonic titers to the test strains were immunized with pneumococcal vaccine; immunization with pneumococcal vaccine induced a titer rise in all but one instance when susceptible GBS strains were tested. In contrast, only a single titer rise was detected when resistant GBS strains were employed in the test. These results indicate that immunization with a cross-reacting antigen (identical to core antigen of type III GBS) fails to induce opsonic antibody to all strains of type III GBS. A resistant strain was made highly susceptible to neutrophil killing in vitro by exposure to neuramindase prior to incubation with opsonic serum. Using a fluorescent lectin-binding assay, this enzyme appeared to remove surface sialic acid, suggesting that sialic acid is an antiphagocytic factor. However, the possibility that other surface moieties may act as antiphagocytic factors cannot be ruled out. Both opsonic susceptible and resistant strains absorbed opsonic antibody from serum, which suggests that the GBS antiphagocytic factors do not prevent binding of antibody to resistant bacteria.

These findings indicate that demonstration of serum opsonic activity to one strain of type III GBS may not accurately depict opsonic activity to other strains. In addition, immunization with core antigen did not enhance opsonic activity against all GBS strains. These data also point out the need to use assays which measure functional antibody, since demonstration of antibody binding may not reflect its ability to facilitate bacterial phagocytosis and killing.

Over the last decade Streptococcus agalactiae, the Group B streptococcus (GBS), has emerged as a major cause of neonatal morbidity and mortality $(1,5,24)$. Progression from colonization to invasive disease seems to involve a complex interaction between the host immune response and the invading GBS. Recent in vitro studies have shown that opsonic antibody is necessary for efficient phagocytosis and killing of GBS $(2,8,16,18,22)$. In addition, type specific antibodies provide protection in experimental mouse (17), suckling rat (10), and chick embryo (23) models of GBS disease. Lancefield $e t$ al. (17) have found that antibodies specific for both carbohydrate and protein determinants on the surface of GBS protect mice from lethal GBS challenge. These data suggest that there may be several antigens on the surface of GBS that react with opsonic antibody and are important in the immune response to these organisms.

Clinical studies have shown that neonates with a deficiency of type specific antibody may be at increased risk to invasive GBS disease $(3,12)$. Using blood transfusions as a means of providing opsonic antibody to babies with GBS sepsis, Shigeoka, et al. (20) have demonstrated that passive administration of opsonic antibody may decrease mortality in neonatal GBS sepsis. It is important to note that survival correlated with the presence of opsonic antibody to the infecting GBS strain.

In previous studies we have reported that type III GBS and Streptococcus pneumoniae type 14 have a similar polysaccharide antigen $(10,11)$ and that cross reacting opsonic antibody is produced to type III GBS by immunizing rabbits with formalinized $S$. pneumonia type $14(10,11)$, or by immunizing humans with pneumococcal vaccine (18). The cross reaction occurs between the hot $\mathrm{HCl}$ extracted antigen (core antigen) of type III GBS and the polysaccharide capsular antigen of type 14 pneumococcus which have an identical chemical composition $(14,15)$. However, the pneumococcal vaccine is not an effective method of providing protection against GBS since only type III strains cross react and there is no evidence that antibody to the other four types would be induced after immunization with pneumococcal vaccine. Furthermore, recent studies by Baker et al. (7) have shown that individuals immunized with pneumococcal vaccine had weaker and less sustained GBS antibody responses than those individuals immunized with GBS antigen containing sialic acid. They also did not detect rises in opsonic antibody to type III GBS in those individuals immunized with pneumococcal vaccine who had lower pre-immunization levels of antibody to type III GBS. The present study further suggests that pneumococcal vaccine would not be an effective method of immunizing women against GBS. In the present study we identify type III GBS resistant to opsonization with human serum containing either naturally occurring or immunization-induced antibodies. Resistant bacteria were susceptible to opsonization after treatment with neuraminidase suggesting that sialic acid may function as an antiphagocytic factor in GBS resistant to opsonization.

\section{MATERIALS AND METHODS}

STREPTOCOCCAL STRAINS

Group B streptococcal strain, IIINor is a clinical isolate cultured from the cerebrospinal fluid of an infant with meningitis (17). Three other type III GBS strains SS893, SS892 and SS891 were kindly supplied by Dr. Hazel Wilkinson, Center for Disease Control, Atlanta, GA. The bacteria were grown to mid log phase in Todd-Hewitt broth (THB), and stored at $-70^{\circ} \mathrm{C}$ until used.

\section{SERUM TREATMENT}

Serum from healthy adults was heat-inactivated at $56^{\circ} \mathrm{C}$ for 30 min, divided into $0.5 \mathrm{ml}$ aliquots and stored at $-70^{\circ} \mathrm{C}$ until thawed for testing. Four individuals whose serum contained undetectable or low titer type III GBS opsonins on initial screening were immunized with pneumococcal vaccine after providing informed consent (Pneumovax, Merck Co., West Point, PA). A venous blood specimen was obtained 6-8 wk after immunization and the serum separated, heat-inactivated and frozen as above. 


\section{ABSORPTION OF SERUM}

Human serum with known opsonic activity was utilized. The bacteria used for absorption were grown overnight on blood agar, scraped from the plate, and suspended in normal saline. The organisms were pelleted in $0.5 \mathrm{ml}$ microfuge tubes to one-fifth the volume of the tube, $0.4 \mathrm{ml}$ of serum was added, the tubes were vortexed and then rotated at slow speed on an end-over-end tumbler (Fisher Rotorack model 343, Fisher Scientific Co., Pittsburg, PA) at $4^{\circ} \mathrm{C}$ overnight. The following day the bacteria were sedimented in the microfuge tube and the supernatant serum was removed and filtered through a $0.2 \mu$ membrane filter. All absorbed sera were used either directly or stored at $-70^{\circ} \mathrm{C}$.

\section{OPSONOPHAGOCYTIC BACTERICIDAL ASSAY}

Opsonization was studied using a microtiter modification (10) of a previously described opsonophagocytic assay (13). Neutrophils from normal adult volunteers were isolated by dextran sedimentation followed by Ficoll-Hypaque density centrifugation. These cells were washed once in $0.85 \%$ saline solution and then resuspended at a concentration of $2.5 \times 10^{7}$ cells $/ \mathrm{ml}$. For the assay, bacteria were grown to mid $\log$ phase in THB, washed once, and then appropriately diluted in Eagle's medium. Ten $\mu \mathrm{l}$ (containing $2.0 \times 10^{4}$ bacteria) were added to round-bottom microtiter wells along with $40 \mu \mathrm{l}$ of the neutrophil suspension, 10 $\mu \mathrm{l}$ of newborn rabbit serum as a source of active complement (screened to ensure the absence of antibody activity against test organisms), and $40 \mu \mathrm{l}$ of varying dilutions of heat-inactivated human serum. Control wells in every experiment consisted of neutrophils alone, complement alone, and neutrophils plus complement. In addition, serum with and without opsonic activity were included as positive and negative controls in each experiment. Cultures with bacteria and antibody plus complement or antibody alone were done periodically to ensure that agglutination was not falsely lowering colony counts. The microtiter plates were sealed and incubated at $37^{\circ} \mathrm{C}$ with constant shaking. To determine neutrophil bactericidal activity, $10 \mu$ l samples were taken from each well at zero time (approximately $5 \mathrm{~min}$ ), and after 1 and $2 \mathrm{~h}$ of incubation. The samples were appropriately diluted in test tubes containing $0.1 \%$ bovine se um albumin in distilled water to selectively lyse the neutrophils; tl ey were then vigorously vortexed to ensure that the bacteria were adequately dispersed before they were cultured on blood agar. Neutrophil bactericidal activity was calculated by the formula: $100-(100 \times A / B)$, where $A$ is the number of bacteria at 60 or $120 \mathrm{~min}$ and $B$ is the number of bacteria in the initial inoculum; $0 \%$ represents bacterial growth. The opsonic titer is considered to be the serum dilution where $80 \%$ or more of the bacteria are nonviable after $\mathbf{2}$ h.

Since this assay was used to detect opsonic antibody directed against GBS and was not concerned with measuring neutrophil function, the neutrophil to bacteria ratio was increased to ensure that all bacteria coated with opsonic antibody would come in contact with an active neutrophil. We have found that this assay is more sensitive in detecting antibody that facilitates phagocytosis and killing of GBS and that killing does not occur in the absence of antibody to GBS.

\section{TREATMENT OF BACTERIA WITH NEURAMINIDASE}

Bacteria were grown to mid $\log$ phase ( 2 to $3 \mathrm{~h}$ ) in THB with the addition neuraminidase ( $1 \mathrm{U} / \mathrm{ml}$ final concentration; from Vibrio cholerae Z4, Grand Island Biological Co., Grand Island, NY). After incubation the bacteria were washed and resuspended in Eagle's media and utilized as described above in the opsonophagocytic bactericidal assay.

\section{LECTIN ANALYSIS OF SURFACE SIALIC ACID}

Limulus Polyphemus Agglutinin (LPA) is a lectin which specifically binds to sialic acid residues. Fluorescein isothiocyanatelabeled LPA, affinity purified and tested for specificity, was obtained from E-Y Laboratories, San Mateo, CA. Aliquots of control and enzyme-treated bacteria $\left(1.0 \times 10^{6}\right.$ bacteria in $\left.0.1 \mathrm{ml}\right)$ were incubated with fluoresceinated LPA at a final concentration of $100 \mu \mathrm{g} / \mathrm{ml}$ for $30 \mathrm{~min}$ at $4^{\circ} \mathrm{C}$. After two washes in cold $0.85 \%$ saline, one drop of bacteria was placed under a coverslip on a microscope slide. Each preparation was examined with a Zeiss Photoscope II with epi-fluorescence, and the \% of bacteria exhibiting surface fluorescence was determined.

\section{RESULTS}

Four GBS type III strains were studied using the opsonophagocytic assay and serum from 15 healthy adults. Strain 893 was highly susceptible to opsonization with all but 2 sera (Table 1); these 2 sera also were not opsonic for any of the GBS strains. Strain IIINor was opsonized by 8 of 15 sera at a dilution of 2.5 or greater, but it was clearly less susceptible to opsonization than 893. In contrast, strains 891 and 892 were resistant to opsonization with most sera $(12 / 15)$. Three of the sera did opsonize strain 891 ( 2 at $\geq 1: 10$ ) suggesting that resistance can be overcome if an appropriate opsonic antibody is present in the serum. Figure 1 shows typical killing curves for strains susceptible (893) and resistant (891) to opsonization. More than $1 \mathrm{log}$ killing is seen at both $1: 2.5$ and 1:5 with 893 , while strain 891 grows in a similar fashion to controls incubated with neutrophils in the absence of antibody.

Four individuals with undetectable or low titers of opsonic antibody to most type III GBS strains were immunized with pneumococcal vaccine that contains an antigen identical to the "core" antigen of GBS type III $(14,15)$. Analysis of pre- and post-immunization sera with the study strains of GBS demonstrated a selective induction of opsonic antibody (Fig. 2). A rise in opsonic titer was observed in all but one instance with postimmunization serum when strains susceptible to opsonization were tested. In contrast, only one vaccinee demonstrated a titer rise against the two resistant strains, suggesting that induction of opsonic antibody with pneumococcal vaccine is to some degree strain specific. A representative assay using susceptible strain 893 (Fig. 3) demonstrated the marked difference in opsonic activity between post-immunization and pre-immunization serum from one vaccinee. Cultures with pre-immunization serum or without serum showed growth. Furthermore, analysis of post-immunization serum showed that there is a marked difference in killing between the opsonization susceptible and opsonization resistant strains. These results clearly demonstrate that pneumococcal immunization of an individual with no detectable natural opsonic antibody to either sensitive or resistant strains may induce opsonic antibody only to the susceptible strains.

To determine if GBS resistance to opsonization was due to sialic acid on the surface, a resistant strain was studied after exposure to neuraminidase. Exposure to neuraminidase made the resistant strain highly susceptible to opsonic antibody without altering the normal growth of the bacteria (Table 2). Furthermore in the absence of opsonic antibody, but in the presence of complement and neutrophils, GBS were still not phagocytized and killed. Sialic acid was detected on the surface of both resistant and susceptible GBS strains using fluorescent-labeled LPA. However, there was not a clear quantitative difference in fluorescence between strains. After incubation with neuraminidase, fluorescence was no longer observed on any GBS, indicating that sialic acid was effectively

Table 1. Opsonic activity of serum from healthy adults for type III Group B streptococci

\begin{tabular}{lrccc}
\hline & \multicolumn{4}{c}{ Reciprocal of opsonic titer } \\
\cline { 2 - 5 } GBS & $<2.5$ & 2.5 & 5.0 & $\geq 10$ \\
strain & $2 / 15$ & $1 / 15$ & $1 / 15$ & $11 / 15$ \\
893 & $7 / 15$ & $2 / 15$ & $2 / 15$ & $4 / 15$ \\
IIINor & $12 / 15$ & $1 / 15$ & $2 / 15$ & \\
892 & $12 / 15$ & $1 / 15$ & & $2 / 15$ \\
891 & & & \\
\hline
\end{tabular}




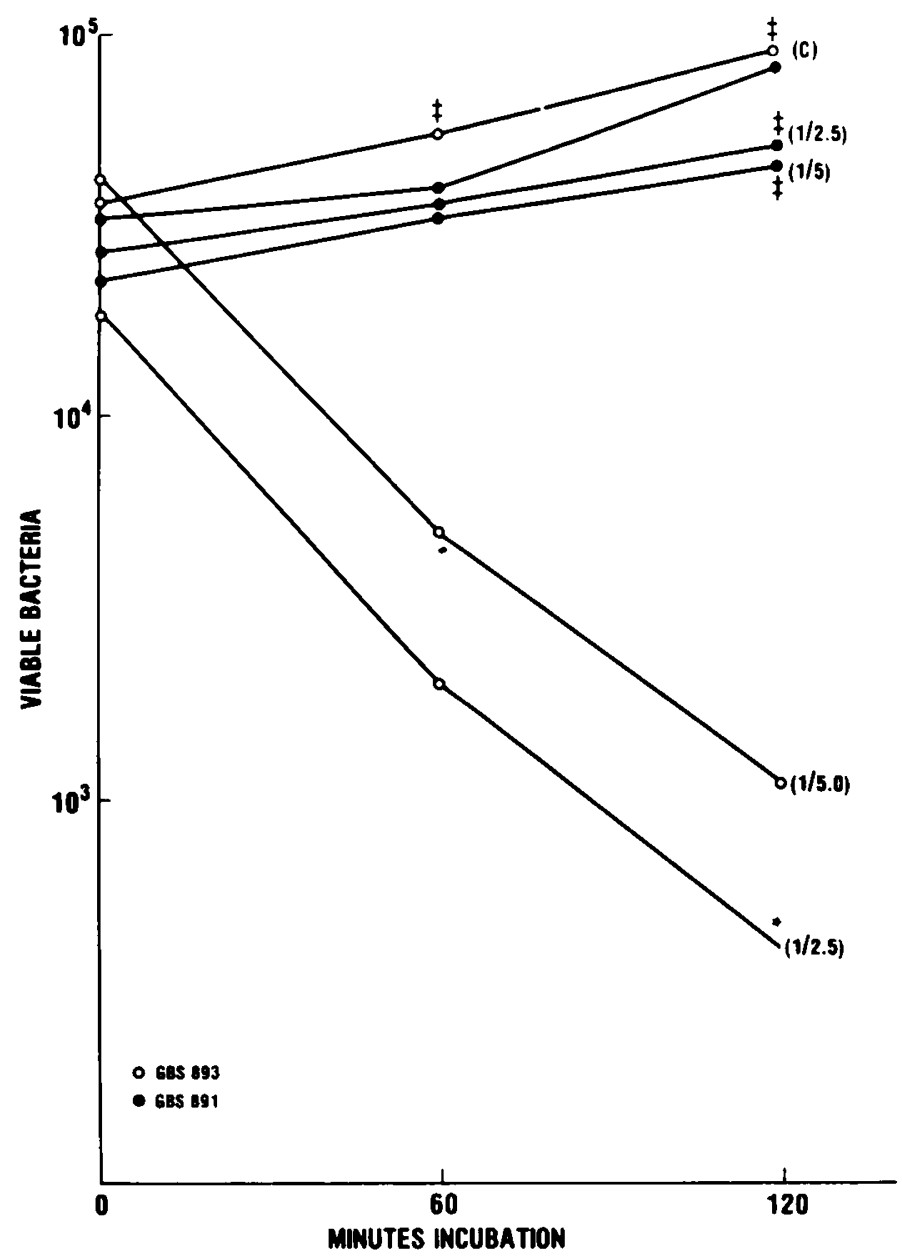

Fig. 1. The effect of human serum on opsonization and killing of Type III GBS. Using serum from an individual not previously immunized with pneumococcal vaccine, susceptible strain 893 showed greater than 1 log killing at serum dilutions of 1:2.5 and 1:5, while the resistant strain 891 showed growth after incubation with the same serum. This growth was similar to control cultures (C) without serum. Control cultures with test serum and complement, but without neutrophils are not shown, but were not different from the other control curves. "No viable bacteria detected (> $2 \log$ killing); ${ }^{++}$At these times, growth was so heavy that accurate quantification above the recorded values was not possible.

removed. These studies do not eliminate the possibility that other moieties on the GBS surface may also inhibit phagocytosis.

To determine whether sialic acid completely masks the galactopyranosyl residue of the "core" antigen, absorption studies were performed with opsonic resistant or susceptible bacteria. Both opsonic resistant and susceptible GBS removed the opsonic antibody to susceptible GBS (Table 3 ), suggesting that sialic acid does not prevent the binding of opsonic antibody to either sensitive or resistant strains.

\section{DISCUSSION}

A clear understanding of the immunologic interaction between GBS and the host is an essential prerequisite to developing effective methods for preventing or treating GBS disease. The present studies identify GBS strains resistant to both natural and immunization-induced opsonic antibody and further show that in these resistant strains, sialic acid is a major factor inhibiting GBS opsonization and killing. GBS strains resistant to opsonization with human serum have been previously identified by Shigeoka, et al. (21), but the present studies differ in two important aspects. First, our strains, resistant to natural opsonins, were found to be resistant to antibody induced by human immunization with a purified polysaccharide antigen identical to the "core" polysac-
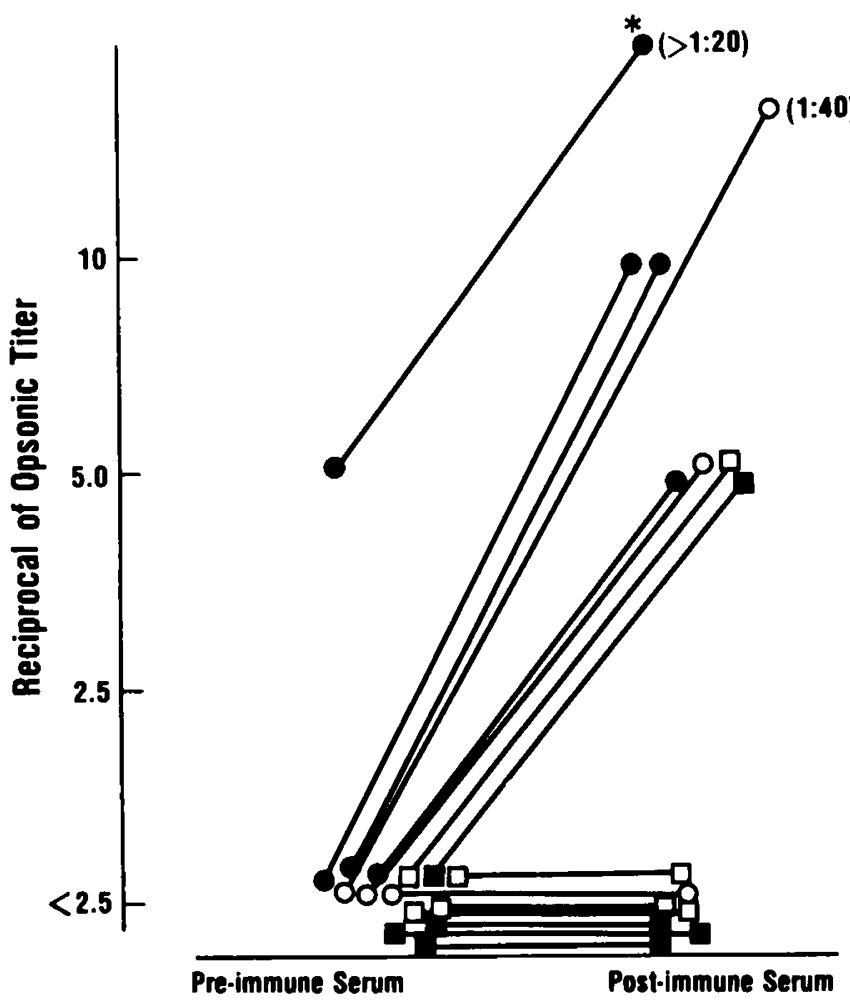

Pre-immune Serum

Post-immune Serum

Oll Nor, 893, 口892, $\square 891$

Fig. 2. The opsonic activity of serum for Type III GBS after immunization of 4 adult volunteers with pneumococcal vaccine. Opsonic activity was generally not detectable in either pre- or post-immunization sera when resistant strains 891 and 892 were used, but was observed in most sera tested against GBS strains susceptible to opsonization (893 and IIINor). Although in these studies $\mathbf{8 0 \%}$ killing is generally used for serum opsonic titer determination, bacterial killing actually exceeded $90 \%$ at the post-immunization titers recorded. *Opsonic antibody to both susceptible strains was present in serum from one vaccine before and after immunization (only 893 is recorded since titers to IIINor are above the scale).

charide antigen found in type III GBS (15). A second difference in our studies is the identification of sialic acid as a specific antiphagocytic factor in resistant strains. In the previous studies trypsin, but not neuraminidase, made resistant strains susceptible to opsonization (21). The type of neuraminidase used in the previous studies may have been incapable of removing GBS sialic acid, which may explain the failure to identify sialic acid as a specific antiphagocytic factor.

Sialic acid has been identified as a component of the type specific antigen of each GBS type (4), though the role of sialic acid in immunity to GBS is not completely understood. Recent studies by Edwards et al. (9) have suggested that sialic acid of type III GBS completely masks end group galactopyranose residues of the core antigen and prevents activation of the alternative complement pathway. In their studies, high levels of antibody to "native" antigen (sialic acid-containing) activated the alternative pathway, while low levels of antibody did not. When they treated the bacteria with neuraminidase the alternative complement pathway was activated and phagocytosis and killing of GBS was accomplished in the absence of antibody. The present studies, using serum from individuals immunized with pneumococcal vaccine as a tool to evaluate antibody to type III GBS core antigen, suggest that sialic acid prevents efficient opsonization of GBS. It has been reported that sialic acid can mask antigenic sites on mouse cells in tissue culture and prevent antibody binding (19). Indeed, when sialic acid was removed from resistant strains, phagocytosis and killing were readily accomplished, but only in the presence of antibody (Table 2). Furthermore, our absorption studies demonstrated that sialic acid does not completely mask 


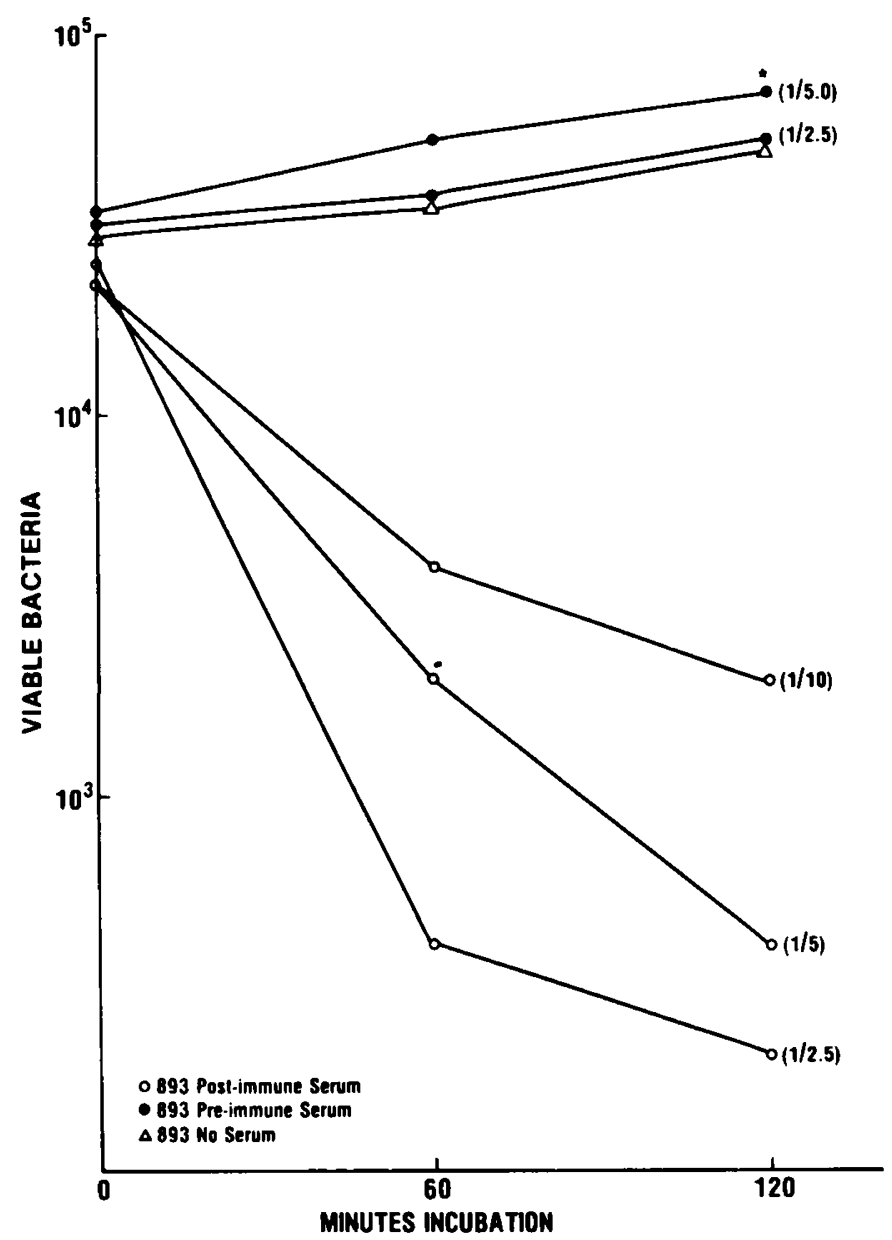

Fig. 3. Comparison of the opsonic activity of human serum for Type III GBS before and after immunization with pneumococcal vaccine. Serum was obtained from an individual before and $8 \mathrm{wk}$ after immunization Cultures with pre-immunization serum or no serum showed an increase in viable GBS (susceptible strain 893), while greater than 1 log killing at several dilutions was observed with post-immunization serum. "core" antigen since the resistant GBS strains bound the opsonic antibody, but were not effectively opsonized and killed until sialic acid was removed by neuraminidase treatment. The present studies also do not eliminate the possibility of other antiphagocytic factors on the surface of GBS.

Although pneumococcal vaccine might provide protection against some type III GBS, infection with a resistant strain like 891 would not be prevented. Furthermore, these studies and the studies of Shigeoka, et al. (21) underscore the importance of using functional assays to determine the presence of opsonic antibody. An antigen binding assay may demonstrate the presence of antibody, but fail to indicate its ability to facilitate phagocytosis and killing of an opsonic resistant strain. Therefore, susceptibility to invasive GBS disease may not be related just to the level of antibody in serum, but also to the characteristics of the invading GBS. Using a radioimmunoassay to measure antibody to GBS, Baker $e$ t al. (6) found mothers of infants with type III disease to have a lower median antibody level as compared to antibody levels in mothers with healthy infants. However, there were wide ranges in antibody concentrations in each group. On the other hand, studies by Wilkinson et al. (25) suggest that radioimmunoassay levels of antibody to GBS antigen are not consistently lower in neonates with GBS infections as compared to uninfected controls. This last observation was true for not only type III, but also other GBS types as well. It seems reasonable to entertain the possibility that infants infected with opsonic resistant strains could have type specific antibody to the infecting GBS, and still be at risk to invasive disease.

Using a functional test such as the opsonophagocytic bactericidal assay to detect antibody to GBS is cumbersome, time consuming and may be less sensitive than other tests such as radioimmunoassay. However, these studies suggest that functional assays may be critical in evaluating GBS antibody. To increase the sensitivity of this assay, the neutrophil ratio was increased to provide a neutrophil excess. As demonstrated in these studies killing did not occur in the absence of serum, (even after neuraminidase treatment of GBS) or in the presence of human serum absorbed with type III GBS.

The identification of type III GBS strains resistant to opsonization with naturally acquired antibody was not surprising, since strains resistant to opsonization had been reported previously. The

Table 2. Opsonic activity of human serum for type III Group B streptococci resistant to opsonic antibody the effect of neuraminidase and trypsin

\begin{tabular}{|c|c|c|c|c|c|c|}
\hline \multirow[b]{2}{*}{ Enzyme } & \multirow{2}{*}{$\begin{array}{l}\text { Serum dilution } \\
\text { (reciprocal) }\end{array}$} & \multicolumn{3}{|c|}{ Viable bacteria $\left(\times 10^{4}\right)$} & \multicolumn{2}{|c|}{ Bactericidal activity } \\
\hline & & $0 \mathrm{~min}$ & $60 \mathrm{~min}$ & $120 \mathrm{~min}$ & $60 \mathrm{~min}$ & $120 \mathrm{~min}$ \\
\hline None & 2.5 & 2.4 & $>4.4$ & $>5.0$ & 0 & 0 \\
\hline None & 5.0 & 3.1 & $>4.1$ & $>5.0$ & 0 & 0 \\
\hline Neuraminidase & 2.5 & 2.4 & 0.3 & 0.04 & $89 \%$ & $98 \%$ \\
\hline Neuraminidase & 5.0 & 3.9 & 0.02 & 0 & $99 \%$ & $100 \%$ \\
\hline None & None & 3.5 & $>4.1$ & $>7.0$ & 0 & 0 \\
\hline Neuraminidase & None & 3.9 & $>5.4$ & $>8.0$ & 0 & 0 \\
\hline
\end{tabular}

Table 3. Removal of opsonic antibody to a susceptible strain of Group B streptococcus ${ }^{1}$ by absorption with strains susceptible or resistant to opsonization

\begin{tabular}{|c|c|c|c|c|c|c|}
\hline \multirow{2}{*}{$\begin{array}{c}\text { Absorbing } \\
\text { strain }\end{array}$} & \multirow{2}{*}{$\begin{array}{l}\text { Serum dilution } \\
\text { (reciprocal) }\end{array}$} & \multicolumn{3}{|c|}{ Viable bacteria $\left(10^{4}\right)$} & \multicolumn{2}{|c|}{ Bactericidal activity } \\
\hline & & $0 \mathrm{~min}$ & $60 \mathrm{~min}$ & $120 \mathrm{~min}$ & $60 \mathrm{~min}$ & $120 \mathrm{~min}$ \\
\hline \multirow[t]{2}{*}{ None } & 2.5 & 4.8 & 2.2 & 0.02 & $55 \%$ & $99 \%$ \\
\hline & 5.0 & 5.2 & 3.1 & 0.1 & $41 \%$ & $99 \%$ \\
\hline \multicolumn{7}{|l|}{891} \\
\hline \multirow[t]{2}{*}{ (Resistant) } & 2.5 & 4.1 & 4.7 & $>8.0$ & 0 & 0 \\
\hline & 5.0 & 4.7 & $>5.5$ & $>8.0$ & 0 & 0 \\
\hline \multicolumn{7}{|l|}{ IIINor } \\
\hline \multirow[t]{2}{*}{ (Susceptible) } & 2.5 & 4.8 & $>5.4$ & $>8.0$ & 0 & 0 \\
\hline & 5.0 & 4.5 & $>5.0$ & $>8.0$ & 0 & 0 \\
\hline None & None & 4.1 & 5.4 & $>6.2$ & 0 & 0 \\
\hline
\end{tabular}

\footnotetext{
1 Test Strain 893.
} 
fact that vaccine-induced antibody was opsonic for some but not all type III GBS strains was unexpected and raises several important considerations concerning immunization to prevent GBS disease. The type 14 antigen in the pneumococcal vaccine is identical to the "core" antigen of type III GBS $(14,15)$. Antisera from individuals immunized with pneumococcal vaccine was used as a probe to study the ability of antibody to "core" antigen to opsonize several strains of type III GBS. These data suggest that surface reactive residues may vary from one GBS strain to another and that sialic acid masking may not always be complete. Sialic acid appears to be an important factor in GBS resistance to phagocytosis and may be an important component of antigens considered for GBS vaccines $(7,9,15)$. However, it is not known if other antiphagocytic factors are present on the surface of GBS which are also capable of rendering certain strains resistant to vaccine-induced opsonic antibody. Further studies are essential to determine the extent and importance of this resistance phenomenon and strain variability. Moreover, the demonstration of antibody to GBS antigens may not be sufficient to assure effective immunity. Protection against invasive GBS disease involves a complex interaction between the bacteria and the host and will require careful evaluation if we are to improve our ability to prevent and treat GBS disease.

\section{REFERENCES AND NOTES}

1. Anthony, B. F. and Okada, D. M.: The emergence of Group B streptococci in infections of the newborn infant. Annu. Rev. Med., 28: 355 (1977).

. Anthony, B. F.: Immunity of Group B streptococci: interaction of serum and macrophages with Types Ia, Ib and Ic. J. Exp. Med., 143: 1186 (1976).

3. Baker, C. J. and Kasper, D. L.: Correlation of maternal antibody deficiency with susceptibility to neonatal Group B streptococcal infection. N. Engl. J. Med. 294: 753 (1976).

4. Baker, C. J. and Kasper, D. L.: Identification of sialic acid in polysaccharide antigens of Group B streptococcus. Infect. Immun., 13: 284 (1976).

5. Baker, C. J.: Group B streptococcal infections: NIH workshop J. Infect. Dis. 136: 137 (1977)

6. Baker, C. J., Kasper, D. L., Tager, I. B., Peredes, A., Alpert, S., McCormack, W. M. and Goroff, D. K.: Quantitative determination of antibody to capsular polysaccharide in infection with Type III strains of Group B streptococcus. J. Clin. Invest, 59: 810 (1977).

7. Baker, C. J., Kasper, D. L., Edwards, M. S. and Schiffman, G.: Influence of preimmunization levels on the specificity of the immune response to related polysaccharide antigens. N. Engl. J. Med., 303: 173 (1980).

8. Baltimore, R. S., Kasper, D. L., Baker, C. J. and Goroff, D. K.: Antigenic specificity of opsonophagocytic antibodies in rabbit antisera to Group B streptococci. J. Immunol., 118: 673 (1977).
9. Edwards, M. S., Nicholson-Weller, A., Baker, C. J. and Kasper, D. L.: The role of specific antibody in alternative complement pathway-mediated opsonophagocytosis of Type III, Group B streptococcus. J. Exp. Med., 151: 1275 (1980).

10. Fischer, G. W., Lowell, G. H., Crumrine, M. H. and Bass, J. W.: Demonstration of opsonic activity and in vivo protection against Group B streptococci Type III by streptococcus pneumoniae Type 14 antisera. J. Exp. Med., 148: 776 (1978).

11. Fischer, G. W., Lowell, G. H., Crumrine, M. H. and Wilson, S. R.: Immunoprecipitation and opsonic cross-reaction between Type 14 pneumococcus and Group B streptococcus Type III. Lancet, l: 75 (1979).

12. Hemming, V. G., Hall, R. T., Rhodes, P. G., Shigeoka, A. O. and Hill, H. R.: Assessment of Group B streptococcal opsonins in human and rabbit serum by neutrophil chemiluminescence. J. Clin. Invest. 58: 1379 (1976).

13. Hirsch, J. G. and Strauss, B.: Studies on heat-labile opsonin in rabbit serum. J. Immunol. 29: 145 (1964).

14. Kasper, D. L., Goroff, D. K. and Baker, C. J.: Immunochemical characterization of native polysaccharides from Group B streptococcus: the relationship of the Type III and Group B determinants. J. Immunol., 121: 1098 (1978).

15. Kasper, D. L., Baker, C. J., Baltimore, R. S., Crabb, J. H., Schiffman, G. and Jennings, H. J.: Immunodeterminant specificity of human immunity to Type III Group B streptococcus. J. Exp. Med., 149: 327 (1979).

16. Klesius, P. H., Zimmerman, R. A., Mathews, J. H. and Krushak, D. H.: Cellular and humoral immune response to Group B streptococci. J. Pediatr., 83: 926 (1973).

17. Lancefield, R. C., McCarty, M. and Everly, W. N.: Multiple mouse-protective antibodies directed against Group B streptococci: special reference to antibodies effective against protein antigens. J. Exp. Med., 142: 165 (1975).

18. Lowell, G. H., Fischer, G. W., Wilson, S. R. and Crumrine, M. H.: Serum from adults immunized with pneumococcal vaccine is opsonic in vitro and protective in vivo for Group B Type III streptococci. Pediatr. Res., 13: 463 (1979).

19. Shearer, W. T., Gottlieb, C. and Kornfeld, S.: Humoral immunostimulation VII. Sialic acid masks antigenic sites on an antibody-selected variant cell line. J. Immunol., 119: 614 (1977)

20. Shigeoka, A. O., Hall, R. T. and Hill, H. R.: Blood transfusions in Group B streptococcal sepsis. Lancet, 1: 636 (1978).

21. Shigeoka, A. O., Hall, R. T. and Hill, H. R.: Strain specificity of opsonins for Group B streptococci Type II and III. Infect. and Immun., 23: 438 (1979).

22. Stewardson-Krieger, P. B., Albrandt, K., Nevin, T., Kretschmer, R. R. and Gotoff, S. P.: Perinatal immunity to Group B hemolytic streptococcus Type la. J. Infect. Dis., I36: 649 (1977)

23. Tieffenberg, J., Vogel, L., Kretschmer, R. R., Padnos, D. and Gotoff, S. P.: Chicken embryo model for type III Group B beta hemolytic streptococcal septicemia. Infect. Immun., 19: 481 (1978).

24. Wilkinson, H. W.: Group B streptococcal infections in humans. Ann. Rev. Microbiol., 32: 41 (1978).

25. Wilkinson, H. W.: Detection of Group B streptococcal antibodies in human sera by radioimmunoassay: concentrations of type-specific antibodies in sera of adults and infants infected with Group B streptococci. J. Clin. Microbiol., 7(2): 194 (1978).

26. Requests for reprints should be addressed to: Dr. Gerald W. Fisher, Uniformed Services University, Department of Pediatrics, Bethesda, MD 20014.

27. Received for publication January $21,1981$.

28. Accepted for publication June 12, 1981. 